\title{
Synthesis of BODIPY-Amino Acids and the Potential Applications as Specific Dyes for the Cytoplasm of Langerhans $\beta$-Cells
}

\author{
Jorge A. Aguilera, ${ }^{a}$ Víctor García-González, ${ }^{b}$ Manuel Alatorre-Meda, ${ }^{c}$ \\ Eustolia Rodríguez-Velázquez $z^{d, e}$ and Ignacio A. Rivero ${ }^{\circledR} *, a$ \\ ${ }^{a}$ Centro de Graduados e Investigación en Química, \\ Tecnológico Nacional de México/Instituto Tecnológico de Tijuana, \\ Blvd. Alberto Limón Padilla S/N 22510 Tijuana, B. C., México \\ ${ }^{b}$ Departamento de Bioquímica, Facultad de Medicina Mexicali, \\ Universidad Autónoma de Baja California, Mexicali, B. C., México \\ ${ }^{c}$ Centro de Graduados e Investigación en Química, Grupo de Biomateriales y Nanomedicina, \\ Cátedras CONACyT-Tecnológico Nacional de México/Instituto Tecnológico de Tijuana, \\ Blvd. Alberto Limón Padilla S/N 22510 Tijuana, B. C., México \\ ${ }^{d}$ Facultad de Odontología, Universidad Autónoma de Baja California, Campus Tijuana, \\ Calzada de Universidad 14418, 22390 Tijuana, B. C., México \\ ${ }^{e}$ Centro de Graduados e Investigación en Química, Grupo de Biomateriales y Nanomedicina, \\ Tecnológico Nacional de México/Instituto Tecnológico de Tijuana, \\ Blvd. Alberto Limón Padilla S/N 22510 Tijuana, B. C., México
}

In this work, we explored the synthesis of 4,4-difluoro-4-bora-3a,4a-diazas-indacene (BODIPYs) bound to five different amino acids (BODIPY-FAA) (glycine, alanine, leucine, phenylalanine, and tyrosine) (amino group is kept protected with fluorophore Fmoc) and evaluated these conjugates in terms of $(i)$ their photophysical properties and (ii) their potential application as cell staining agents of suspension and adherent cells at healthy and stress conditions. In general, all synthesized BODIPY-FAA (3a-3e) were found to emit fluorescence in the blue and green regions of the spectrum (depending on the solvent conditions). However, BODIPY-FTyr(trt) (3e) showed the best molar extinction coefficient $\left(\varepsilon=28,198 \mathrm{M}^{-1} \mathrm{~cm}^{-1}\right)$ and quantum yield $(\Phi=0.17)$. Biologically speaking, all synthesized conjugates demonstrated a selective affinity for the cytoplasm of Langerhans $\beta$-cells employed as a model, being the BODIPY-FLeu conjugate the one displaying the highest observed intensity. As such, our results reveal the BODIPY-FAA as a novel attractive tool for the specific staining of the cell cytoplasm, demonstrating not only a dual fluorescence emission but also a sensing capability to recognize different cell states.

Keywords: BODIPY-amino acids dyes, bio-conjugation, cell staining

\section{Introduction}

Amino acids are one of the most important blocks in biological processes. ${ }^{1}$ Moreover, amino acids are natural inducers of structural specificity at the intracellular level since their interaction with cell surfaces produces different and selective results, likewise, amino acids are molecules that are recognized by the biological system and are biocompatible. Among others, they are involved in the

*e-mail: irivero@tectijuana.mx formation of peptides and proteins, and in the course of any aberrant alteration in protein folding at a physiological level often manifested in common metabolic disorders, serious neurological conditions such as Alzheimer and Parkinson diseases, as well as cardiovascular diseases, ${ }^{2,3}$ Branchedchain, ${ }^{4}$ aminoaciduria, glutaric acidurias, phenylketonuria, nonketotic hyperglycinemia, homocystinuria, etc. The ascertaining of the L-amino acids in a biological system is of great importance since they are an essential part of the metabolism of proteins and physiology. The amino acids found in the intestine, especially those with 
aromatic rings such as tryptophan and phenylalanine, are determining factors in the absorption of proteins given that they participate in the release of gastric acid ${ }^{5-7}$ and pancreatic enzymes. ${ }^{8,9}$ However, the endocrine tissues, the intestinal physiological processes as well as the specific receptors involved in the detection of amino acids are not completely characterized. ${ }^{10}$ Therefore, the elucidation of the intracellular tracking and performance of amino acids at healthy and disease conditions is believed to be pivotal for the understanding of several biological processes not completely elucidated nowadays.

Cell internalization and the biological role of amino acids such as leucine, arginine, and glutamine have been studied in recent publications ${ }^{11-13}$ with the help of diverse cellular and liposomal models. From a biochemical point of view, amino acids have high selectivity in a biological system, mainly for the chirality of their asymmetric center (L), or axial (helical) chirality. However, the monitoring and characterization of amino acids at the intracellular level is challenging given that the abundance of endogenous amino acids in the intracellular compartments does not allow them to be differentiated from those that are added to investigate their mobility and residence in cellular systems. Also, amino acids do not have very efficient optical properties and can generate a residual emission that does not favor their tracking or location. Therefore, amino acids and short peptides have to be modified with synthetic molecules to favor their selfassembly and tracking inside cells. Derivatives of chiral amino acids linked to aromatic molecules by click synthesis are of great interest for their photophysical properties such as absorption and emission (fluorescence) with potential biological recognition of proteins (bovine serum albumin (BSA)) and sugars (arabinose). ${ }^{14,15}$

With respect to the promotion of their self-assembly, an illustrative example of a modification for this purpose is the functionalization of amino acids with 9-fluorenylmethyloxycarbonyl (Fmoc), a protection group of the amino end. Fmoc promotes the self-assembly of the protected amino acids through hydrophobic interactions. ${ }^{16}$ On the other hand, the insertion of fluorescent dyes into the backbone of amino acids can lead to the development of macromolecules possessing biomimetic characteristics, with unique structural and biological properties by which they can be easily detected by different spectroscopic and microscopy techniques. ${ }^{17,18}$ Amino acids have a wide functional diversity due to structural variations in their side chain, those that contain chromophore groups in this chain are very important for their potential applications. ${ }^{19-21}$ In this context, one feasible strategy for the tracking and characterization of amino acids at the extracellular and intracellular levels is its coupling to fluorescent probes, such as 4,4-difluoro-4-bora-3a,4a-diazas-indacene (BODIPY). This has been used as a fluorescent probe in the determination of hypochlorous acid $(\mathrm{HOCl}) /$ hypochlorite (OCl) which play an important role in the immune system, ${ }^{22,23}$ also a BODIPY-nitroolefin was prepared for the determination of small molecule bio-thiols, including cysteine (Cys), homocysteine (Hcy), and glutathione (GSH), which play many crucial roles in physiological processes. ${ }^{24}$ Likewise, the combination of a BODIPY with $p$-hydroxybenzaldehyde to determine the hydrogen sulfide plays an important role in the health and development of various diseases. $^{25}$

In 2010, Peña-Cabrera and co-workers ${ }^{26}$ reported the synthesis of 8-propargylamine-BODIPY (8-PAB), which emits in the blue region with high quantum yields $(\Phi)\left(\lambda_{\text {max emiss }}=469.5 \mathrm{~nm}, \Phi=0.94\right.$, ethyl acetate $)$. The conventional BODIPY dyes usually emit at a longer wavelength $(\lambda)$, above $500 \mathrm{~nm}$, and up to $900 \mathrm{~nm}$, depending on further conjugation. ${ }^{27}$ Subsequent studies disclosed that related 8-amino-BODIPY dyes, readily prepared from 8-(thiomethyl)-BODIPY, ${ }^{28}$ consistently emit in the blue region with high quantum yields $\left(\lambda_{\text {max emiss }}=444-470 \mathrm{~nm}\right.$, $\Phi=0.42-1.0$, ethyl acetate). More recently, other authors have reported the use of BODIPY-8-ethylenediamine (BODIPY-EDM) as a fluorescence resonance energy transfer (FRET) system and for cyclization and biological labeling (Scheme 1). ${ }^{17,29-31}$

In general, BODIPY has been developed a lot and have had applications in various areas of knowledge, ${ }^{32}$ such as medicine and biotechnology. ${ }^{33}$ These are organic optoelectronic materials and have been applied in bioimaging technology, ${ }^{34}$ as a sensor and sensitizing agent in cancer phototherapy, ${ }^{35}$ etc.

In this work, we describe a novel and highly reproducible method of synthesis of BODIPY bound to five different amino acids (BODIPY-FAA) coupled to five different amino acids such as Fmoc-glycine (FGly), Fmoc-alanine (FAla), Fmoc-leucine (FLeu), Fmoc-phenylalanine (FPhe), and Fmoc-tyrosine (FTyr), which are protected with the Fmoc group, increasing the lipophilicity (Scheme 2). The obtained conjugates were characterized in terms of their photophysical properties and staining performance in vitro upon culture with suspension and adherent cells under healthy and stressful conditions.

\section{Experimental}

\section{General procedures}

All reagents and solvents were purchased from a commercial source (Sigma-Aldrich, Saint Louis, USA) 
<smiles>NCCNC1=C2C=CC=[N+]2B(F)n2cccc21</smiles><smiles>O=C(O)c1ccc(N2C(=O)C=CC2=O)cc1</smiles>

Kim et al. ${ }^{17}$<smiles>C=COc1ccc2c(c1)Oc1cc(OC=C)ccc1C21OC(=O)c2cc(NS(=O)(=O)[O-])ccc21</smiles><smiles></smiles><smiles>CC(=S)NCCNC1=C2C=CC=[N+]2B(F)n2cccc2C1=C1C=CC=[N+]1B(F)F</smiles>

$\mathrm{HgCl}_{2}$<smiles>FB1n2cccc2C(N2CCN=C2Nc2ccccc2)=C2C=CC=[N+]12</smiles>

Wang et al. ${ }^{30}$<smiles>CC1=C2C=CC=[N+]2B(F)n2cccc21</smiles><smiles>O=C1NCCN1C1=C2C=CC=[N+]2B(F)B(F)n2cccc21</smiles>

Scheme 1. Different synthesis with BODIPY-EDM..$^{1729-31}$<smiles></smiles>

(5)<smiles></smiles>

(6)

$\mathrm{R}$; - $\mathrm{H}$ (Fmoc-Gly), - $\mathrm{CH}_{3}$ (Fmoc-Ala), - $\mathrm{CH}_{2}-\mathrm{CH}\left(\mathrm{CH}_{3}\right)_{2}$, (Fmoc-leu), $-\mathrm{CH}_{2}-\mathrm{C}_{6} \mathrm{H}_{5}$, (Fmoc-Phe), $-\mathrm{CH}_{2}-\mathrm{C}_{6} \mathrm{H}_{4}-\mathrm{O}-\mathrm{C}\left(\mathrm{CH}_{3}\right)_{3}$, (Fmoc-Tyr-O-Trt)

Scheme 2. A general method to synthesize the BODIPY-FAA.

and used without further purification. Anhydrous solvents for organics synthesis were prepared by passing through a solvent purification tower. Silica gel 60 (230-400 mesh) was used to purify the products. Thin-layer chromatography (TLC) was performed on silica gel $\mathrm{F}_{254}$ plates (Sigma-
Aldrich, Saint Louis, USA). All compounds were detected using UV light. Melting points were obtained with an Electrothermal 88629 apparatus and were not corrected. Fourier transform infrared spectra (FTIR) were recorded with a PerkinElmer FT-IR 1600 spectrometer. ${ }^{1} \mathrm{H},{ }^{13} \mathrm{C}$, and 
${ }^{19} \mathrm{~F}$ nuclear magnetic resonance (NMR) spectra at 400, 101, and $376 \mathrm{MHz}$ were recorded, respectively, using a Bruker Avance III spectrometer in $\mathrm{CDCl}_{3}$, dimethyl sulfoxide (DMSO- $d_{6}$ ), or $\mathrm{CD}_{3} \mathrm{OD}$ with tetramethylsilane (TMS) as an internal standard, and trifluoroacetic acid was the reference for ${ }^{19} \mathrm{~F}$ spectra. Mass spectra were obtained with an Agilent Technologies 5975C MS spectrometer at $70 \mathrm{eV}$ by direct insertion and an Agilent HPLC (Mod 1100) coupled to mass selective detector (MSD) version SL. High-resolution mass spectra (HRMS) were obtained with an Agilent ESIQTOF (Mod G6530B). UV-Vis absorption spectra were obtained with a Varian Cary 50 SCAN spectrophotometer. Fluorescence spectra were recorded with a Photon Technology International Fluorescence System with a $1 \mathrm{~cm}$ standard quartz cell.

\section{Method to prepare BODIPY-FAA}

BODIPY-8 ethylenediamine (BODIPY-EDM) was prepared by modification of the literature procedure, ${ }^{17}$ and the synthetic routes to produce the BODIPY-FAA derivatives are outlined in Scheme 3.

\section{Synthesis of BODIPY-EDM}

\section{Di(1H-pyrrol-2-yl)methanethione (2)}

Tiophosgene $(0.46 \mathrm{~mL}, 6.03 \mathrm{mmol})$ in toluene $(10 \mathrm{~mL})$ at $0{ }^{\circ} \mathrm{C}$ was added to solution of pyrrole $(0.81 \mathrm{~g}$, $12.07 \mathrm{mmol})$ in ethyl ether $(15 \mathrm{~mL})$. After $10 \mathrm{~min}$, it was added a solution of $\mathrm{CH}_{3} \mathrm{OH}-\mathrm{H}_{2} \mathrm{O}(9: 1)$ (12 mL). The mixture was stirred by $30 \mathrm{~min}$ at room temperature (RT). The solvent excess was removed by reduced pressure and chromatographed with neutral alumina and eluted with petroleum ether:dichloromethane (8:2), to get a crystal red solid. $0.65 \mathrm{~g}, 65 \%$; ${ }^{1} \mathrm{H} \mathrm{NMR}\left(200 \mathrm{MHz}, \mathrm{CDCl}_{3}\right) \delta 6.40$ (m, 2H, CH), 7.04 (m, 2H, CH), 7.16 (s, 2H, CH), 9.77 (s, $1 \mathrm{H}) ;{ }^{13} \mathrm{C} \mathrm{NMR}\left(50 \mathrm{MHz}, \mathrm{CDCl}_{3}\right) \delta 112.0,114.5,127.6$, 138.3, 193.0.

(E)-2-((Methylthio)(2H-pyrrol-2-ylidene)methyl)-1H-pyrrole (3)

Methyl iodide ( $5 \mathrm{~mL}, 45 \mathrm{mmol})$ in dichloromethane was added to a solution of $2(0.880 \mathrm{~g}, 5.0 \mathrm{mmol})$, the reaction mixture was stirring $24 \mathrm{~h}$ at RT. The excess of solvent was eliminated to reduced pressure to get a brown oil. The crude was used in the next step without further purification (684 $\mathrm{mg}, 65 \%) ;{ }^{1} \mathrm{H} \mathrm{NMR}\left(200 \mathrm{MHz}, \mathrm{CDCl}_{3}\right) \delta 2.89$ (s, 3H), 6.64-6.63 (m, 2H), 7.24-7.23 (m, 2H), 7.88-7.87 (m, 2H), $12.01(\mathrm{~s}, 1 \mathrm{H}) ;{ }^{13} \mathrm{C} \mathrm{NMR}\left(50 \mathrm{MHz}, \mathrm{CDCl}_{3}\right) \delta 21.5,116.7$, 128.6, 129.3, 138.4, 162.2.

\section{5,5-Difluoro-10-(methylthio)- $5 H-4 \lambda^{4}, 5 \lambda^{4}$-dipyrrolo} [1,2-c:2',1'-f][1,3,2]diazaborinine (BODIPY-MeS) (4)

$\mathrm{BF}_{3} \cdot \mathrm{OEt}_{2}(6 \mathrm{~mL}, 45 \mathrm{mmol})$ was added drop by drop to a solution of 3 (1.18 g, $6.21 \mathrm{mmol}), \mathrm{Et}_{3} \mathrm{~N}$ ( $\left.5 \mathrm{~mL}, 30 \mathrm{mmol}\right)$ in dichloromethane $(20 \mathrm{~mL})$. The mixture reaction was stirred by $24 \mathrm{~h}$. After, the excess of solvent was removed by reduced pressure. The crude was chromatographed in silica gel and

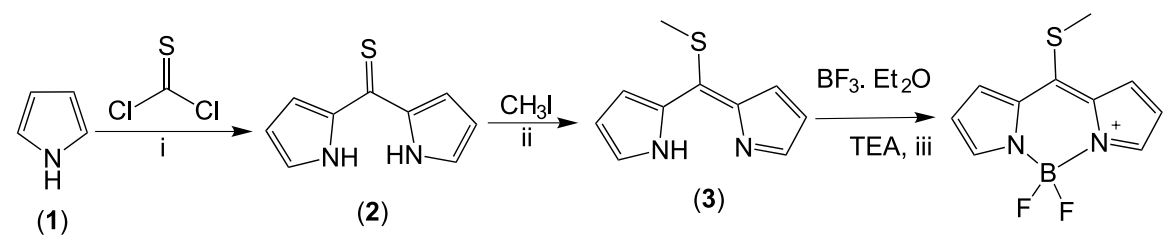

(1)

(2)

(4)

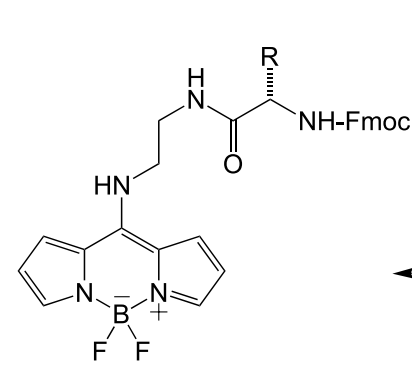

(6)

$\mathrm{R} ;(6 \mathbf{a})-\mathrm{H}$ (Fmoc-Gly), (6b) $-\mathrm{CH}_{3}$ (Fmoc-Ala),

(6c) $-\mathrm{CH}_{2}-\mathrm{CH}\left(\mathrm{CH}_{3}\right)_{2}$, (Fmoc-leu),

(6d) $-\mathrm{CH}_{2}-\mathrm{C}_{6} \mathrm{H}_{5}$, (Fmoc-Phe),

(6e) $-\mathrm{CH}_{2}-\mathrm{C}_{6} \mathrm{H}_{4}-\mathrm{O}-\mathrm{C}\left(\mathrm{CH}_{3}\right)_{3}$, (Fmoc-Tyr-O-Trt)
Conditions: i) $40 \mathrm{~min}, \mathrm{RT}$. ii) $24 \mathrm{~h}$, RT. iii) 24 h, RT. iv) 2 h, RT.

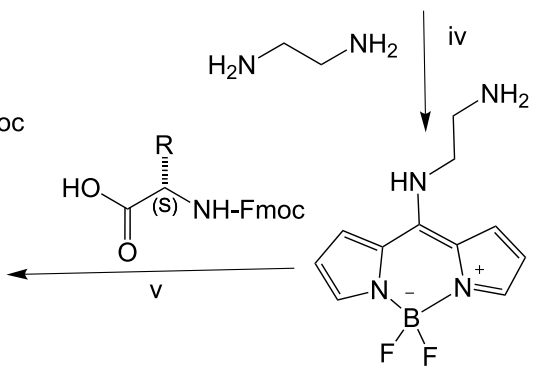

(5)

Scheme 3. Synthesis steps of BODIPY-FAA. 
it was eluted with petroleum ether:ethyl acetate (17:3) to get a dark red solid. $0.89 \mathrm{~g}, 71 \%$; UV-Vis (ACN) $\lambda / \mathrm{nm} 527$ $\left(\varepsilon 40,000 \mathrm{M}^{-1} \mathrm{~cm}^{-1}\right)$; FTIR (attenuated total reflectance (ATR), neat) $v / \mathrm{cm}^{-1} 1264\left(v_{\mathrm{C}-\mathrm{N}}\right), 1489\left(v_{\mathrm{N}}^{+}\right) ;{ }^{1} \mathrm{H} \mathrm{NMR}(200 \mathrm{MHz}$, $\left.\mathrm{CDCl}_{3}\right) \delta 3.20(\mathrm{~s}, 3 \mathrm{H}), 6.93-6.72(\mathrm{~m}, 2 \mathrm{H}), 7.71(\mathrm{~d}, J 4.3 \mathrm{~Hz}$, $2 \mathrm{H}), 8.09$ (s, 2H); ${ }^{13} \mathrm{C} \mathrm{NMR}\left(50 \mathrm{MHz}, \mathrm{CDCl}_{3}\right) \delta 20.0,117.6$, 127.3, 133.4, 140.1, 154.0; ${ }^{19} \mathrm{~F}$ NMR $\left(188 \mathrm{MHz}, \mathrm{CDCl}_{3}\right) \delta$ $-145.6,-145.7,-145.8,-145.9$.

$N^{1}$-(5,5-Difluoro-5H-4 $\lambda^{4}, 5 \lambda^{4}$-dipyrrolo[1,2-c:2', 1'-f][1,3,2]diazaborinin-10-yl)ethane-1,2-diamine (5)

Ethylenediamine $(15 \mathrm{mg}, 0.21 \mathrm{mmol})$ was added to solution of BODIPY-MeS $(50 \mathrm{mg}, 0.21 \mathrm{mmol})$ in dichloromethane $(5 \mathrm{~mL})$ and stirred for $2 \mathrm{~h}$. The excess of solvent was eliminated to reduced pressure and the crude was recrystallized in ethanol to get a yellow solid; (53 mg, 95\%); UV-Vis (ACN) $\lambda / \mathrm{nm} 404\left(\varepsilon\right.$ 37,685 $\left.\mathrm{M}^{-1} \mathrm{~cm}^{-1}\right)$; molecular fluorescence (MF) (ACN) $\lambda / \mathrm{nm} 453 ;{ }^{1} \mathrm{H} \mathrm{NMR}$ $\left(200 \mathrm{MHz}, \mathrm{CDCl}_{3}\right) \delta 3.16-3.10(\mathrm{t}, J 4.2 \mathrm{~Hz}, 2 \mathrm{H}), 3.65-3.59$ (t, J 4.2 Hz, 2H), 6.40 (s, 1H), 6.49 (s, 1H), 6.98 (s, 1H), 7.09 (s, 1H), $7.48(\mathrm{~s}, 1 \mathrm{H}), 7.68(\mathrm{~s}, 1 \mathrm{H}) ;{ }^{13} \mathrm{C} \mathrm{NMR}(50 \mathrm{MHz}$, $\left.\mathrm{CDCl}_{3}\right) \delta 40.0,49.9,111.5,113.0,113.8,120.5,123.8$, 127.3, 128.1, 148.9, 130.1; ${ }^{19} \mathrm{~F} \mathrm{NMR}\left(188 \mathrm{MHz} \mathrm{CDCl}_{3}\right) \delta$ $-146.31,-146.46,-146.62,-146.77$.

\section{General method for preparing BODIPY-FAA (6a-6e)}

$N^{\prime}$-Ethyl carbodiimide hydrochloride (100 mg, $0.524 \mathrm{mmol})$, hydroxy benzotriazole $(70 \mathrm{mg}, 0.524 \mathrm{mmol})$, $N$-methyl morpholine $(144 \mu \mathrm{L}, 1.308 \mathrm{mmol})$ and the corresponding amino acid $(0.48 \mathrm{mmol})$ were added to a solution of BODIPY-EDM (94 $\mathrm{mg}, 0.436 \mathrm{mmol})$ in anhydrous dichloromethane $(8 \mathrm{~mL})$. The reaction was stirred for $5 \mathrm{~h}$ under argon at room temperature $\left(25^{\circ} \mathrm{C}\right)$. The solvent was evaporated under reduced pressure. Purification by flash column chromatography (9:1, dichloromethane/ ethyl acetate) yielded $\mathbf{6 a - 6 e}$ as a yellow solid.

(9H-Fluoren-9-yl)methyl(2-((2-)((5,5-difluoro-5H-4 $\lambda^{4}, 5 \lambda^{4}$ dipyrrolo[1,2-c:2,'1'-f][1,3,2] diaza borinin-10-yl)amino)ethyl) amino)-2-oxoethyl)carbamate (6a)

$134 \mathrm{mg}, 67 \%$; $\mathrm{mp} 165-170{ }^{\circ} \mathrm{C}$; UV-Vis (ACN) $\lambda / \mathrm{nm}$ 394 ( $\varepsilon 13,089 \mathrm{M}^{-1} \mathrm{~cm}^{-1}$ ); MF (ACN) $\lambda / \mathrm{nm} 444$ (Ф 0.15 in ACN); FTIR (ATR, neat) $v / \mathrm{cm}^{-1} 1643\left(v_{\mathrm{C}=\mathrm{C}}\right), 1731\left(v_{\mathrm{C}=\mathrm{O}}\right)$; ${ }^{1} \mathrm{H}$ NMR (400 MHz, DMSO- $d_{6}$ ) $\delta 3.56$ (q, $\left.J 6.1 \mathrm{~Hz}, 2 \mathrm{H}\right)$, $3.68(\mathrm{~d}, J 6.0 \mathrm{~Hz}, 2 \mathrm{H}), 3.84$ (t, J 6.3 Hz, 2H), 4.33-4.17 (m, $3 \mathrm{H}), 6.45-6.32$ (m, 1H), 6.57 (dd, J 4.1, $2.2 \mathrm{~Hz}, 1 \mathrm{H}), 7.44-$ $7.23(\mathrm{~m}, 5 \mathrm{H}), 7.47-7.40(\mathrm{~m}, 1 \mathrm{H}), 7.52(\mathrm{~d}, J 4.2 \mathrm{~Hz}, 1 \mathrm{H})$, 7.76-7.55 (m, 4H), 7.93-7.79 (m, 2H), $8.46(\mathrm{t}, J 5.8 \mathrm{~Hz}$, $1 \mathrm{H}), 9.64(\mathrm{~s}, 1 \mathrm{H}) ;{ }^{13} \mathrm{C}$ NMR (101 MHz, DMSO- $\left.d_{6}\right) \delta 37.6$, 39.3, 39.5, 39.7, 39.9, 40.1, 40.3, 40.5, 44.0, 47.0, 47.6,
$66.2,113.4,114.9,116.9,120.5,122.2,123.9,125.5$, $125.7,127.5,128.1,131.1,134.2,141.2,144.2,148.8$, 157.0, 171.5; ${ }^{19} \mathrm{~F}$ NMR (376 MHz, DMSO- $\left.d_{6}\right) \delta-142.7$ (dd, $J$ 57.5, $25.7 \mathrm{~Hz}$ ); ESI-MS $m / z, \mathrm{C}_{28} \mathrm{H}_{26} \mathrm{BF}_{2} \mathrm{~N}_{5} \mathrm{O}_{3}$, found: $527.80\left(\mathrm{M}^{-}\right)$; HRMS (ESI) $m / z$, calcd. for $\mathrm{C}_{28} \mathrm{H}_{26} \mathrm{BF}_{2} \mathrm{~N}_{5} \mathrm{NaO}_{3}$ $[\mathrm{M}]^{+}:$552.1989, found: 552.1987.

(9H-Fluoren-9-yl)methyl(S)-(1-((2-((5,5-difluoro-5H-4 $\lambda^{4}, 5 \lambda^{4}-$ dipyrrolo[1,2-c:2,'1'-f][1,3,2] diazabo-rinin-10-yl)amino)ethyl) amino)-1-oxopropan-2-yl)carbamate (6b)

$153 \mathrm{mg}, 75 \%$; mp $155-160{ }^{\circ} \mathrm{C}$; UV-Vis (ACN) $\lambda / \mathrm{nm}$ 394 ( $\varepsilon 22,436 \mathrm{M}^{-1} \mathrm{~cm}^{-1}$ ); MF (ACN) $\lambda / \mathrm{nm} 444$ (Ф 0.11 in ACN); FTIR (ATR, neat) $v / \mathrm{cm}^{-1} 1632\left(v_{\mathrm{C}=\mathrm{C}}\right), 1715\left(v_{\mathrm{C}=\mathrm{O}}\right)$; ${ }^{1} \mathrm{H}$ NMR (400 MHz, DMSO- $\left.d_{6}\right) \delta 3.57$ (q, J $\left.6.3 \mathrm{~Hz}, 2 \mathrm{H}\right)$, 3.85 (q, J $6.0 \mathrm{~Hz}, 2 \mathrm{H}), 4.13-4.01(\mathrm{~m}, 1 \mathrm{H}), 4.30-4.14(\mathrm{~m}$, $3 \mathrm{H}), 6.43-6.33(\mathrm{~m}, 1 \mathrm{H}), 6.60-6.54(\mathrm{~m}, 1 \mathrm{H}), 7.38$ (dq, J 26.1, $7.6 \mathrm{~Hz}, 6 \mathrm{H}), 7.53$ (d, J 4.1 Hz, 1H), 7.78-7.60 (m, 4H), 7.89 $(\mathrm{d}, J 7.6 \mathrm{~Hz}, 2 \mathrm{H}), 8.53(\mathrm{t}, J 5.9 \mathrm{~Hz}, 1 \mathrm{H}), 9.75(\mathrm{t}, J 5.8 \mathrm{~Hz}$, $1 \mathrm{H}) ;{ }^{13} \mathrm{C}$ NMR (101 MHz, DMSO- $\left.d_{6}\right) \delta 18.4,40.8,47.0$, 47.8, 50.6, 66.1, 113.4, 114.9, 116.8, 120.5, 122.2, 123.9, $125.5,125.7,125.7,127.5,128.1,131.1,134.2,141.1$, 144.2, 144.3, 148.8, 156.2, 175.0; ${ }^{19} \mathrm{~F}$ NMR (376 MHz, DMSO- $\left.d_{6}\right) \delta-142.8$ (dd, $J$ 59.0, 26.0 Hz); ESI-MS $\mathrm{m} / z$, $\mathrm{C}_{29} \mathrm{H}_{28} \mathrm{BF}_{2} \mathrm{~N}_{5} \mathrm{O}_{3}$, found: $541.75\left(\mathrm{M}^{-}\right)$; HRMS (ESI) $\mathrm{m} / z$, calcd. for $\mathrm{C}_{29} \mathrm{H}_{28} \mathrm{BF}_{2} \mathrm{~N}_{5} \mathrm{NaO}_{3}[\mathrm{M}]^{+}:$566.2145, found: 566.2145.

(9H-Fluoren-9-yl)methyl(S)-(1-((2-)(5,5-difluoro-5H-4 $\lambda^{4}, 5 \lambda^{4}-$ dipyrrolo[1,2-c:2,'1'-f][1,3,2] diaza borinin-10-yl)amino)ethyl) amino)-4-methyl-1-oxopentan-2-yl)carbamate (6c)

$160 \mathrm{mg}, 73 \%$; mp $178-182{ }^{\circ} \mathrm{C}$; UV-Vis (ACN) $\lambda / \mathrm{nm}$ 394 ( $\varepsilon$ 34,638 $\mathrm{M}^{-1} \mathrm{~cm}^{-1}$ ); MF (ACN) $\lambda / \mathrm{nm} 444$ (Ф 0.13 in $\mathrm{ACN})$; FTIR (ATR, neat) $v / \mathrm{cm}^{-1} 1632\left(v_{\mathrm{C}=\mathrm{C}}\right), 1700\left(v_{\mathrm{C}=\mathrm{O}}\right)$; ${ }^{1} \mathrm{H}$ NMR (400 MHz, DMSO- $\left.d_{6}\right) \delta 0.85(\mathrm{dd}, J 15.6,6.4 \mathrm{~Hz}$, $6 \mathrm{H}), 1.66-1.39(\mathrm{~m}, 2 \mathrm{H}), 3.61-3.51(\mathrm{~m}, 2 \mathrm{H}), 3.84(\mathrm{q}, J 4.9 \mathrm{~Hz}$, 2H), 4.10-3.99 (m, 1H), 4.38-4.16 (m, 3H), 6.36 (dd, J 3.9, $2.3 \mathrm{~Hz}, 1 \mathrm{H}), 6.56$ (dd, J 4.1, 2.2 Hz, 1H), 7.46-7.27 (m, 7H), 7.59-7.48 (m, 2H), 7.64-7.56 (m, 2H), $7.72(\mathrm{t}, J 8.5 \mathrm{~Hz}, 2 \mathrm{H})$, 7.88 (d, J 7.6 Hz, 2H), 8.52 (t, J 5.8 Hz, 1H), $9.71(\mathrm{~s}, 1 \mathrm{H})$; ${ }^{13} \mathrm{C}$ NMR (101 MHz, DMSO- $\left.d_{6}\right) \delta 21.9,23.3,24.6,37.7$, 41.0, 47.1, 47.8, 53.5, 66.0, 113.4, 114.8, 116.8, 120.5, 122.2, 123.9, 125.5, 125.7, 127.4, 128.0, 131.1, 134.2, 141.1, 144.1, 144.3, 148.8, 156.4, 174.8; ${ }^{19} \mathrm{~F}$ NMR (376 MHz, DMSO- $\left.d_{6}\right) \delta-142.7(\mathrm{dd}, J 57.5,25.7 \mathrm{~Hz}$ ); ESI-MS $\mathrm{m} / \mathrm{z}$, $\mathrm{C}_{32} \mathrm{H}_{34} \mathrm{BF}_{2} \mathrm{~N}_{5} \mathrm{O}_{3}$, found: $583.80\left(\mathrm{M}^{-}\right)$; HRMS (ESI) $\mathrm{m} / \mathrm{z}$, calcd. for $\mathrm{C}_{32} \mathrm{H}_{34} \mathrm{BF}_{2} \mathrm{~N}_{5} \mathrm{NaO}_{3}[\mathrm{M}]^{+}$: 608.2615 , found: 608.2619.

(9H-Fluoren-9-yl)methyl(S)-(1-((2-)(5,5-difluoro-5H-4 $\lambda^{4}, 5 \lambda^{4}$ dipyrrolo[1,2-c:2,1'-f][1,3,2] diazabo rinin-10-yl)amino)ethyl) amino)-1-oxo-3-phenylpropan-2-yl)carbamate (6d)

$140 \mathrm{mg}, 60 \%$; mp $178-182{ }^{\circ} \mathrm{C}$; UV-Vis (ACN) $\lambda / \mathrm{nm}$ 394 ( $\varepsilon$ 14,703 $\mathrm{M}^{-1} \mathrm{~cm}^{-1}$ ); MF (ACN) $\lambda / \mathrm{nm} 444$ (Ф 0.18 in 
$\mathrm{ACN})$; FTIR (ATR, neat) $v / \mathrm{cm}^{-1} 1654\left(v_{\mathrm{C}=\mathrm{C}}\right), 1705\left(v_{\mathrm{C}=\mathrm{O}}\right)$; ${ }^{1} \mathrm{H}$ NMR (400 MHz, DMSO- $\left.d_{6}\right) \delta 2.87$ (dd, $J 13.6,9.5 \mathrm{~Hz}$, $1 \mathrm{H}), 3.00$ (dd, $J 13.6,5.4 \mathrm{~Hz}, 1 \mathrm{H}), 3.48$ (dd, $J 13.6,6.5 \mathrm{~Hz}$, $1 \mathrm{H}), 3.59$ (dq, J 11.9, $5.8 \mathrm{~Hz}, 1 \mathrm{H}), 3.75$ (s, 2H), 4.23-4.09 (m, 3H), 4.31-4.19 (m, 1H), 6.38 (dd, J 3.9, $2.3 \mathrm{~Hz}, 1 \mathrm{H})$, 6.58 (dd, J 4.2, 2.2 Hz, 1H), 7.24-7.09 (m, 3H), 7.36-7.22 (m, 4H), 7.45-7.35 (m, 4H), 7.49 (d, J 4.1 Hz, 1H), 7.69$7.59(\mathrm{~m}, 3 \mathrm{H}), 7.74(\mathrm{~d}, J 8.3 \mathrm{~Hz}, 1 \mathrm{H}), 7.87$ (d, J 7.6 Hz, 2H), $8.57(\mathrm{t}, J 5.8 \mathrm{~Hz}, 1 \mathrm{H}), 9.68(\mathrm{~s}, 1 \mathrm{H}) ;{ }^{13} \mathrm{C} \mathrm{NMR}(101 \mathrm{MHz}$, DMSO- $\left.d_{6}\right) \delta 37.6,37.9,40.9,47.0,47.7,56.7,66.1,113.4$, $114.9,116.8,120.5,122.2,123.9,125.5,125.7,125.7$, $126.7,127.4,127.5,128.0,128.5,129.6,131.1,134.3$, 138.2, 141.1, 144.2, 144.2, 148.8, 156.2, 173.7; ${ }^{19} \mathrm{~F}$ NMR (376 MHz, DMSO- $d_{6}$ ) $\delta-142.8$ (dd, $J$ 58.2, $25.5 \mathrm{~Hz}$ ); ESI-MS $m / z, \mathrm{C}_{35} \mathrm{H}_{32} \mathrm{BF}_{2} \mathrm{~N}_{5} \mathrm{O}_{3}$, found: $617.85\left(\mathrm{M}^{-}\right)$; HRMS (ESI) $\mathrm{m} / z$, calcd. for $\mathrm{C}_{35} \mathrm{H}_{32} \mathrm{BF}_{2} \mathrm{~N}_{5} \mathrm{NaO}_{3}[\mathrm{M}]^{+}$: 642.2458, found: 642.2465 .

(9H-Fluoren-9-yl)methyl(S)-(3-(4-(tert-butoxy)phenyl)1-((2-((5,5-difluoro-5H-4 $\lambda^{4}, 5 \lambda^{4}$-dipyrrolo[1,2-c:2', 1'-f][1,3,2] diazaborinin-10-yl)amino)ethyl)amino)-1-oxopropan-2-yl) carbamate (6e)

$148 \mathrm{mg}, 57 \%$; mp $128-132{ }^{\circ} \mathrm{C}$; UV-Vis (ACN) $\lambda / \mathrm{nm}$ 394 ( $\varepsilon 28,198 \mathrm{M}^{-1} \mathrm{~cm}^{-1}$ ); MF (ACN) $\lambda / \mathrm{nm} 444$ (Ф 0.17 in ACN); FTIR (ATR, neat) $v / \mathrm{cm}^{-1} 1651\left(v_{\mathrm{C}=\mathrm{C}}\right), 1703\left(v_{\mathrm{C}=\mathrm{O}}\right)$; ${ }^{1} \mathrm{H}$ NMR (400 MHz, DMSO- $d_{6}$ ) $\delta 1.15$ (s, 9H), 2.81 (dd, $J$ 13.6, $9.5 \mathrm{~Hz}, 1 \mathrm{H}), 2.96$ (dd, J 13.6, $5.4 \mathrm{~Hz}, 1 \mathrm{H}), 3.54$ (ddt, $J 43.9,13.5,6.2 \mathrm{~Hz}, 2 \mathrm{H}), 3.75$ (d, J 8.9 Hz, 2H), 4.28-4.06 (m, 4H), 6.58 (dd, J 4.2, $2.2 \mathrm{~Hz}, 1 \mathrm{H}), 6.80-6.73$ (m, 2H), 7.18-7.11 (m, 2H), 7.36-7.24 (m, 2H), 7.46-7.35 (m, 4H), $7.51(\mathrm{~d}, J 4.2 \mathrm{~Hz}, 1 \mathrm{H}), 7.74-7.59$ (m, 3H), 7.78 $(\mathrm{d}, J 8.4 \mathrm{~Hz}, 1 \mathrm{H}), 7.88(\mathrm{~d}, J 7.6 \mathrm{~Hz}, 2 \mathrm{H}), 8.62(\mathrm{t}, J 5.8 \mathrm{~Hz}$, $1 \mathrm{H}), 9.75(\mathrm{~s}, 1 \mathrm{H}) ;{ }^{13} \mathrm{C}$ NMR $\left(101 \mathrm{MHz}, \mathrm{DMSO}-d_{6}\right) \delta 28.9$, 37.3, 37.6, 47.0, 47.7, 56.7, 66.2, 78.0, 113.5, 114.9, 116.8, $120.5,122.2,123.7,124.0,125.5,125.8,127.5,128.0$, $130.1,131.1,132.7,134.3,141.1,144.2,148.7,153.9$, 156.2, 173.8; ${ }^{19} \mathrm{~F}$ NMR (376 MHz, DMSO- $\left.d_{6}\right) \delta-142.7$ (dd, $J$ 57.5, 25.7 Hz); ESI-MS, $m / z \mathrm{C}_{39} \mathrm{H}_{40} \mathrm{BF}_{2} \mathrm{~N}_{5} \mathrm{O}_{4}$, found: $689.88\left(\mathrm{M}^{-}\right)$; HRMS (ESI) $m / z$, calcd. for $\mathrm{C}_{39} \mathrm{H}_{40} \mathrm{BF}_{2} \mathrm{~N}_{5} \mathrm{NaO}_{4}$ $[\mathrm{M}]^{+}:$714.3034, found: 714.3042 .

\section{Preparation of small unilamellar vesicles (SUVs)}

SUVs composed of phosphatidylcholine (PC) and PC-cholesterol were prepared according to a previously reported protocol. ${ }^{36}$ Lipids were mixed in chloroform and dried for 90 min under a gentle stream of $\mathrm{N}_{2}$ with an additional incubation of $5 \mathrm{~h}$ at $25^{\circ} \mathrm{C}$ in a Speed Vac concentrator (Savant). After drying, the samples were hydrated in buffer $\mathrm{pH} 7.4$ and afterward processed through 4 cycles of freezing in liquid $\mathrm{N}_{2}$, and thawing at $37^{\circ} \mathrm{C}$, and finally a sonication process (10 min for cycles of $15 \mathrm{~s}$ on/30 s off at 9.5-10 watts) in an Ultrasonic Homogenizer. Samples were incubated $2 \mathrm{~h}$ at $25{ }^{\circ} \mathrm{C}$ and then centrifuged for $10 \mathrm{~min}$ at 13,000 rpm. The obtained solution of SUVs was kept at $4{ }^{\circ} \mathrm{C}$ until use. Then, SUVs were incubated with different BODIPY-amino acids for $12 \mathrm{~h}$, and then the emission spectra of BODIPY-amino acid conjugates were recorded in the range of 440-540 nm, using an excitation wavelength of $425 \mathrm{~nm}$.

\section{Cell culture}

The established rat $\beta$-cell line RIN-m5F (American Type Culture Collection, ATCC) was grown in Roswell Park Memorial Institute Medium (RPMI)-1640. Hepatocyte cultures (C9-derived rat liver cells, ATCC) were grown in Dulbecco's Modified Eagle Medium (DMEM). For both cultures, $10 \%$ fetal bovine serum (FBS) was used, supplemented with $10 \mathrm{U} \mathrm{mL}^{-1}$ penicillin, $10 \mu \mathrm{g} \mathrm{mL} \mathrm{m}^{-1}$ streptomycin, and $25 \mu \mathrm{g} \mathrm{mL}^{-1}$ amphotericin $\mathrm{B}$. The cell cultures were maintained at $37{ }^{\circ} \mathrm{C}$ in a humidified atmosphere of $5 \% \mathrm{CO}_{2}$. The culture medium was changed every 3 to 4 days and the cultures were passaged once a week.

\section{Fatty acid preparation}

Models of lipotoxicity were evaluated according to the background of our group. ${ }^{37}$ Palmitic (PA), oleic acid (OA), and palmitoleic acid (PAO) stock solutions were prepared in an ethanol: $\mathrm{H}_{2} \mathrm{O}$ solution $(1: 1 ; \mathrm{v}: \mathrm{v})$ at $60{ }^{\circ} \mathrm{C}$ to reach a final concentration of $75 \mathrm{mM}$. Stock solutions were complexed with fatty-acid-free bovine serum albumin (BSA) ( $3 \mathrm{mM}$ in $\mathrm{H}_{2} \mathrm{O}$ ) by stirring for $1.5 \mathrm{~h}$ at $37^{\circ} \mathrm{C}$, then diluted in culture media and filtered (filter pore size of $0.22 \mu \mathrm{m}$ ). The final molar ratio of fatty acid:BSA was $3: 1$ with a final concentration of $300 \mu \mathrm{M}$ of BSA.

Preparation of lipopolysaccharides (LPS)

LPS O111:B4 was diluted in ultrapure $\mathrm{H}_{2} \mathrm{O}$ to a concentration of $1 \times 10^{6} \mathrm{ng} \mathrm{mL} \mathrm{mL}^{-1}$, sonicated for $10 \mathrm{~min}$, and filtered (filter pore size of $0.22 \mu \mathrm{m}$ ), to be subsequently diluted in culture medium to different concentrations, according to a stablished protocol. ${ }^{38}$

\section{Confocal microscopy}

A LEICA TCS-SP8 confocal microscope (LEICA Microsystems Heidelberg $\mathrm{GmbH}$ ) was employed to characterize the cellular distribution of the BODIPY- 
amino acids $(8 \mu \mathrm{M})$ under different metabolic overload conditions. For these experiments, cells were proliferated to reach $90 \%$ of confluence on glass coverslips, previously treated with UV radiation for $30 \mathrm{~min}$. Then, during $1 \mathrm{~h}$, cells were maintained in starving conditions without phosphate-buffered saline (PBS) and treated under different experimental conditions. Later, cell cultures were washed with PBS $1 \times$, incubated for 30 min with BODIPY-amino acids, then Hoechst dye was added (when necessary) to the cultures and incubated for $30 \mathrm{~min}$. Finally, cultures were washed twice with cold PBS $1 \times$, fixed with paraformaldehyde, and mounted for observation. Macroscopically different zones were recorded, preferentially at the center of the specimens, to depict representative images. The images were recorded at excitation/emission wavelengths of 405/430-550 (for detection of Hoechst, blue channel) and 488/495-545 (for detection of BODIPY-amino acids, green channel).

\section{Results and Discussion}

\section{Chemical synthesis of BODIPY-amino acids}

The synthesis of the BODIPY-amino acid conjugates from different protected amino acids was achieved by the route described in Scheme 1. In this work, we used five amino acids: Fmoc-Gly-OH, Fmoc-Ala-OH, Fmoc-Leu-OH, Fmoc-Phe-OH, and Fmoc-Tyr(trt)-OH, three of which are aliphatic and two with aromatic properties. The synthesis began with the binding of BODIPY-EDM to the corresponding protected amino acids, in accordance with a reported methodology for peptide coupling, ${ }^{17}$ to obtain the 6a-6e derivatives with different reaction yields from 57 to $75 \%$.

\section{Photophysical properties}

The experimental UV-Vis absorption and fluorescence emission spectra of the BODIPY-amino acid conjugates (6a-6e) (1 to $10 \mu \mathrm{M})$ were acquired in acetonitrile (Supplementary Information (SI) section Figures S33-S36). Figure 1 depicts representative spectra for the BODIPY-FLeu system. This figure reveals that Fmoc-Leu-OH and BODIPYEDM spectra have distinctive absorption and emission bands that are present in the BODIPY-FLeu one. This finding demonstrates the conjugation of the protected amino acid with BODIPY-EDM to form BODIPY-FLeu. The spectra of the other conjugates are shown in the SI section.

The BODIPY-amino acid conjugates (6a-6e) show the main absorption band at $394 \mathrm{~nm}$ and a molar extinction coefficient in a range of 13,089 to $34,638 \mathrm{M}^{-1} \mathrm{~cm}^{-1}$
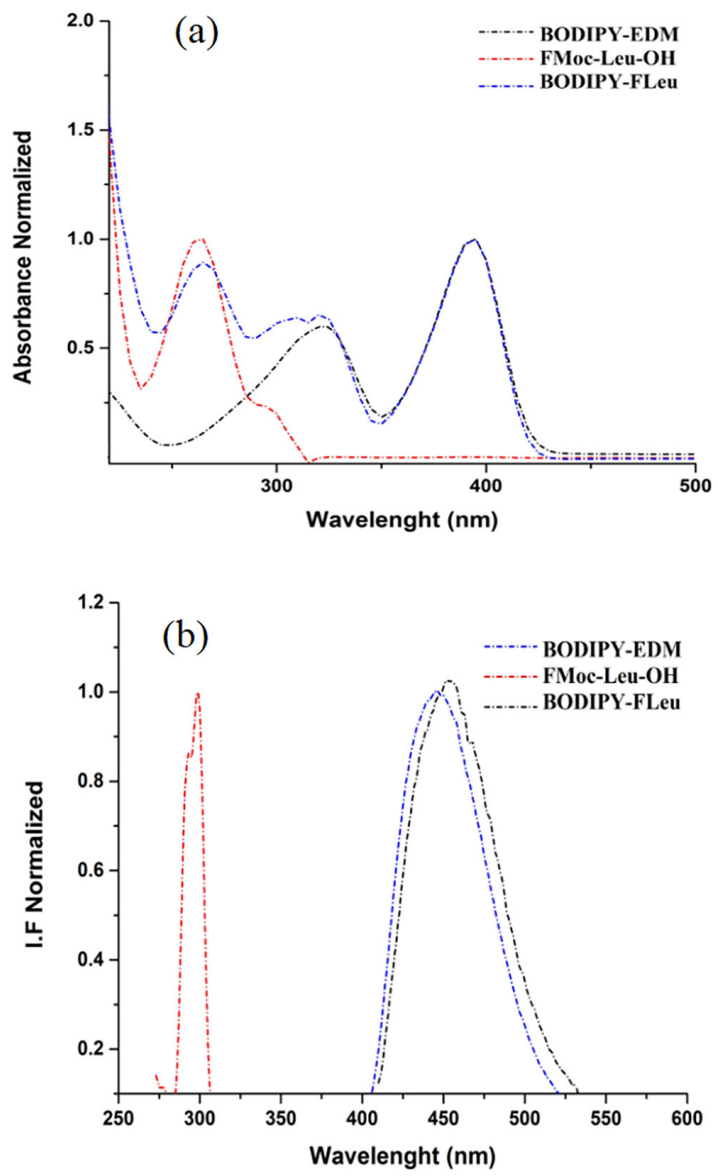

Figure 1. (a) UV-Vis spectra of BODIPY-FLeu, of BODIPY-EDM, and Fmoc-Leu-OH, and (b) fluorescence intensity (FI) of BODIPY-FLeu $\left(\lambda_{\max \text { abs }} 394 \mathrm{~nm}, \lambda_{\text {max emiss }} 444 \mathrm{~nm}\right)$, BODIPY-EDM $\left(\lambda_{\max \text { abs }} 394 \mathrm{~nm}\right.$, $\lambda_{\max \text { emiss }} 444 \mathrm{~nm}$ ), and Fmoc-Leu-OH ( $\left.\lambda_{\max \text { abs }} 264 \mathrm{~nm}, \lambda_{\max \text { emiss }} 296 \mathrm{~nm}\right)$. The spectra were acquired in acetonitrile.

(Table 1). For the different BODIPY's, there is no change in the absorption band that is independent of the amino acid; however, the molar extinction coefficient was found to be dependent on the side chain of the amino acids. Peaks observed at the highest energies can be assigned to spin-allowed $\pi-\pi^{*}$ transitions of aromatic $(260 \mathrm{~nm})$ and fluorenyl $(298 \mathrm{~nm})$ groups. The BODIPYs with aliphatic amino acids showed molar extinction coefficients as good as the aromatics, in particular, the BODIPY-FLeu was the one with the highest observed coefficient.

The studies carried out by molecular fluorescence showed that there are two emitting units in the BODIPY-amino acid conjugates: the Fmoc and the BODIPY group, detected at ca. 300 and $444 \mathrm{~nm}$, respectively. However, the unit governing the emission process is the BODIPY (Figure 1) since the strongest band is observed at $444 \mathrm{~nm}$, which is attributed to $\mathrm{S}_{0}-\mathrm{S}_{1}$ BODIPY transition. This band located in the blue region of the spectrum (see Table 1) resulted from excitation with maximum absorption of $394 \mathrm{~nm}$. Interestingly, higher quantum yields were observed for the 
Table 1. Photophysical properties of BODIPY-FAA dyes

\begin{tabular}{lccccc}
\hline BODIPY-FAA & $\lambda_{\max }$ abs $/ \mathrm{nm}$ & $\varepsilon /\left(\mathrm{M}^{-1} \mathrm{~cm}^{-1}\right)$ & $\lambda_{\max \text { emiss }} / \mathrm{nm}$ & $\Delta$ Stokes $/ \mathrm{nm}$ & $\Phi^{\mathrm{a}}$ \\
\hline BODIPY-FGly & 394 & 13,089 & 444 & 50 & 0.15 \\
BODIPY-FAla & 394 & 22,436 & 444 & 50 & 0.11 \\
BODIPY-FLeu & 394 & 34,638 & 444 & 50 & 0.13 \\
BODIPY-FPhe & 394 & 14,703 & 444 & 50 & 0.18 \\
BODIPY-FTyr(trt) & 394 & 28,198 & 444 & 50 & 0.17 \\
\hline
\end{tabular}

a-PAB in acetonitrile as standard $(\Phi=0.94) .{ }^{26}$ BODIPY-FAA: 4,4-difluoro-4-bora-3a,4a-diazas-indacene bound to five different amino acids; $\lambda$ : wavelength; $\varepsilon$ : molar extinction coefficient; $\Phi$ : quantum yield; FGly: Fmoc-glycine; FAla: Fmoc-alanine; FLeu: Fmoc-leucine; FPhe: Fmoc-phenylalanine; FTyr: Fmoc-tyrosine; Fmoc: 9-fluorenylmethyloxycarbonyl.

conjugates containing amino acids with aromatic groups such as Phe and Tyr(trt), indicating that amino acids increase the planarity of the molecule an increase in the quantum yield (Table 1). This effect can be considered as advantageous for the use of amino acids containing aromatic groups as sensing compounds at the extracellular level. However, amino acids with aliphatic chains are less polar and their aliphatic tail could interact more selectively with the components of the cell membrane and the cytoplasm inside cells. For instance, leucine, the amino acid with the largest chain under study is very feasible to increase its interaction by promoting a more efficient fixation with molecules of the plasmatic membrane and organelle membranes in the cytoplasm, such as phospholipids. In this sense, a branched-chain amino acid (BCAA) such as isoleucine, valine, and leucine show the highest positive values of the hydropathic index: 4.5, 4.2, and 3.8, respectively. ${ }^{38}$ Hydropathy values reflect the free energy $(\Delta G)$ of transference of the lateral chain of amino acids from a hydrophobic solvent to water. Then, Leu is an amino acid with a high propensity to interact with hydrophobic biomolecules (hydropathy index 3.8), contrary to Gly (hydropathy index -0.4 ).

As already discussed, the photophysical properties of the BODIPY-amino acid conjugates were characterized in acetonitrile. The 8-PAB was the standard used to obtain the relative quantum yields and the value of 0.94 corresponds to the study carried out in acetonitrile. Therefore, our studies to determine the relative quantum yields were in acetonitrile and it is because the polar aprotic solvents avoid the formation of hydrogen bond interactions between the solute and the solvent. ${ }^{26,39}$ Fluorescence studies were carried out both in aqueous solution and with PBS, no difference in emission was found in both solutions.

However, additional studies were carried out to determine the water effect on the fluorescence emission of the BODIPYamino acid conjugates. This effect is very important since the study in cells had to be performed in an aqueous solution. Figure 2 shows the UV spectrum of BODIPYFLeu in acetonitrile and water. A slight bathochromic shift of the absorption bands was observed when using water as a solvent. Previous studies ${ }^{40}$ show that this effect is proportional to the polarity and viscosity of the solvent.

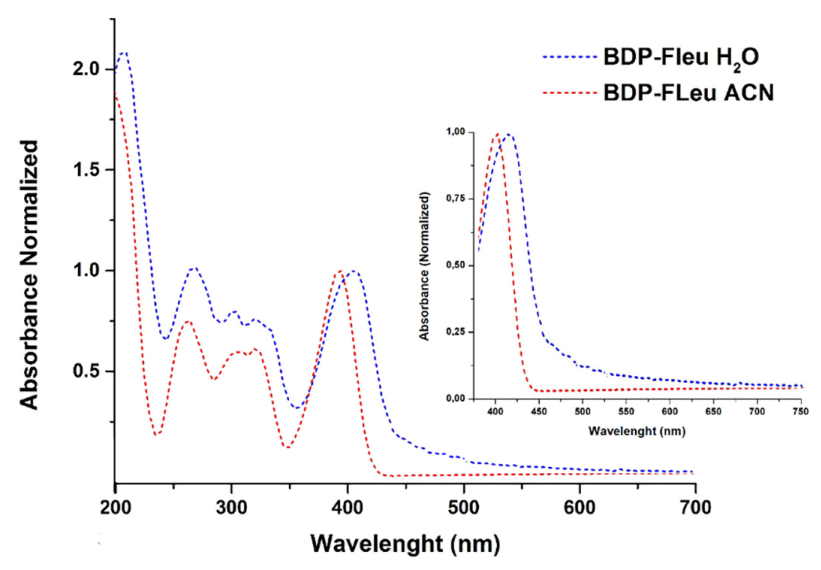

Figure 2. UV spectrum of BODIPY-FLeu in solvents as acetonitrile and water.

Interestingly, this shift opens the possibility of acquiring confocal micrographs of the system either at the blue or the green channels, which proves to be important considering that common staining agents for cell nuclei emit in blue, such as Hoechst and 6-diamidino2-phenylindole (DAPI). As discussed below, we employed BODIPY-FLeu in the absence and presence of Hoechst to stain $\beta$-cells for the confocal microscopy characterization. The obtained images revealed an outstanding contrast of both dyes, demonstrating that the fluorescence emission of BODIPY-FLeu can be tuned on demand by varying the wavelength of the excitation laser: $405 \mathrm{~nm}$ to obtain images in blue (images not shown) and $488 \mathrm{~nm}$ to obtain images in green (when used in combination with blueemitting dyes as shown in the presented images).

\section{Experimentation with small unilamellar vesicles (SUV's)}

To evaluate the binding of the BODIPY-amino acid conjugates to specific biomolecules or cellular structures, in a first approach we used SUVs as a model of lipid membranes. The emission spectra of the different BODIPY- 
amino acids were recorded in the range of 440-540 $\mathrm{nm}$, using an excitation wavelength of $425 \mathrm{~nm}$.

Experiments revealed an evident change in the emission spectra of the BODIPY-amino acids upon interaction with SUV's composed of phosphatidylcholine (PC) and PC plus cholesterol (PC-Chol). Specifically, the interaction of both SUV systems with BODIPY-FPhe and SUV-BODIPY-FLeu led to a marked decrease of fluorescence of the BODIPY-amino acids (Figures 3a, 3b), suggesting possible intercalation of the amino acids into the PC bilayer membrane due to the hydrophobic nature of the side chains, originating a quenching effect, that could be related to dynamics of the lipid bilayer. Indeed, in the characterization of phosphatidylcholine/cholesteryl-esters liposomes, we demonstrated a lipid ordering phenomenon modulated by the C-terminus domain of cholesteryl-ester transfer protein (CETP) in which hydrophobic amino acids were intercalated in the hydrophilic/hydrophobic interface of phospholipids. ${ }^{41}$ By contrast, incubation of both SUVs with BODIDY-FGly gave rise to an increase in the fluorescence emission of the BODIPY amino acid, which is likely to be associated with the hydrophilic nature of the amino acid (glycine) (Figure 3c).

\section{Characterization upon cell internalization}

Based on the previous results, the following step in this study was the characterization of cell internalization and intracellular performance of the BODIPY-amino acids. To this end, hepatocytes (C9 cells) and Langerhans $\beta$-cells were tested in suspension and adherent conditions, respectively. First, hepatocytes were proliferated up to a confluence of $90 \%$ and retrieved in a cellular suspension for incubation with the BODIPY-amino acid conjugates. Figure 4 shows the data of fluorescence emission obtained upon the culture of the cells with BODIPY-FPhe (Figure 4a), BODIPY-FLeu (Figure 4b), and BODIPY-FGly (Figure 4c). Very interestingly, it can be observed from this figure that the three samples under study exhibited an increase in fluorescence emission when internalized by cells, being BODIPY-FLeu (Figure 4b) the one rendering the best result. This phenomenon could be associated with greater interaction with phospholipids of the cytoplasmic membrane and membrane-bound organelles, such as the endoplasmic reticulum (ER) or mitochondria.

\section{Confocal microscopy of stained Langerhans $\beta$-cells}

Langerhans $\beta$-cells were chosen as a model for the characterization at adherent conditions. This cell line is of human physiopathological relevance associated
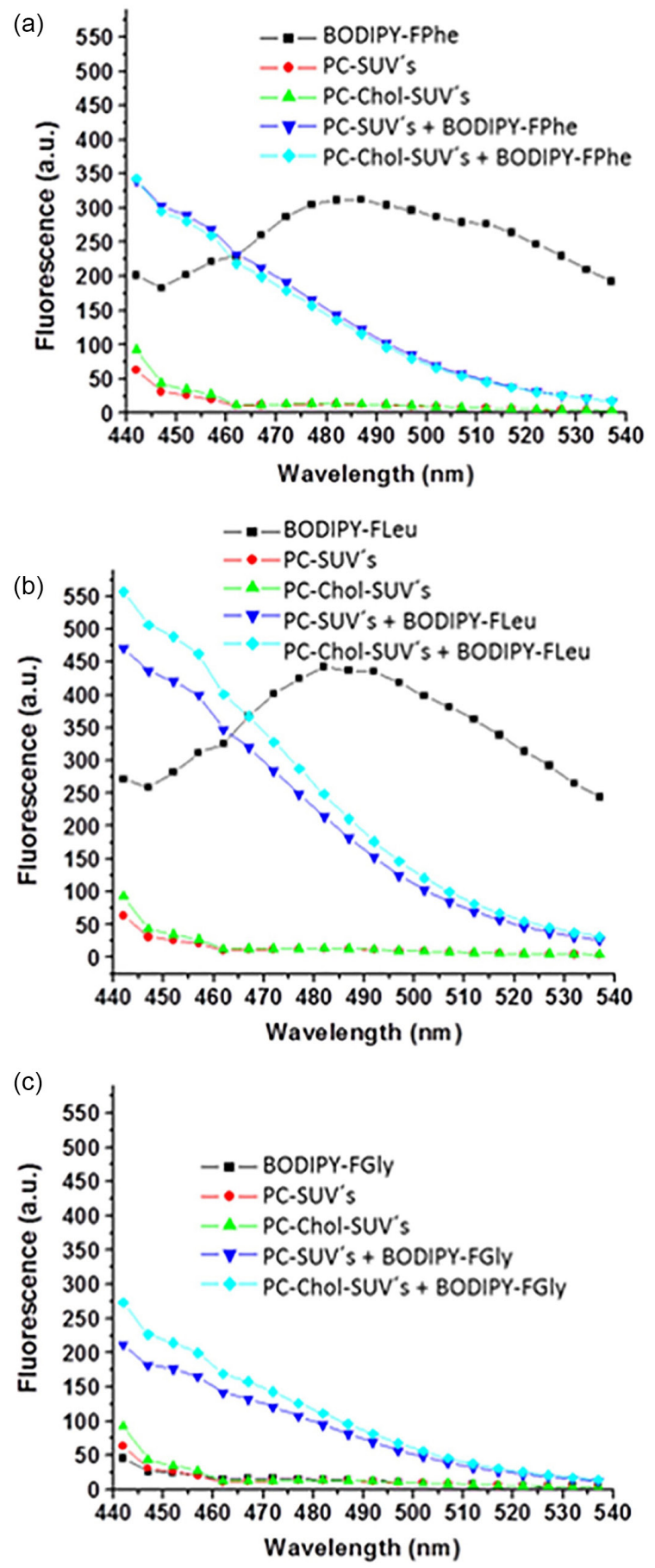

Figure 3. Fluorescence spectra of BODIPY-amino acid conjugates in the absence and presence of PC and PC-Chol SUV's.

with metabolic overload, namely diabetes mellitus type- $2,{ }^{42}$ being a model in which we have performed the characterization of mechanisms related with calcium regulation and ER stress. ${ }^{43,44}$

The five probes were evaluated for Langerhans cell staining and the results are shown in Figure 5. This figure depicts that all BODIPY-amino acids have a high selectivity for the cytoplasm region (green channel), rendering an 

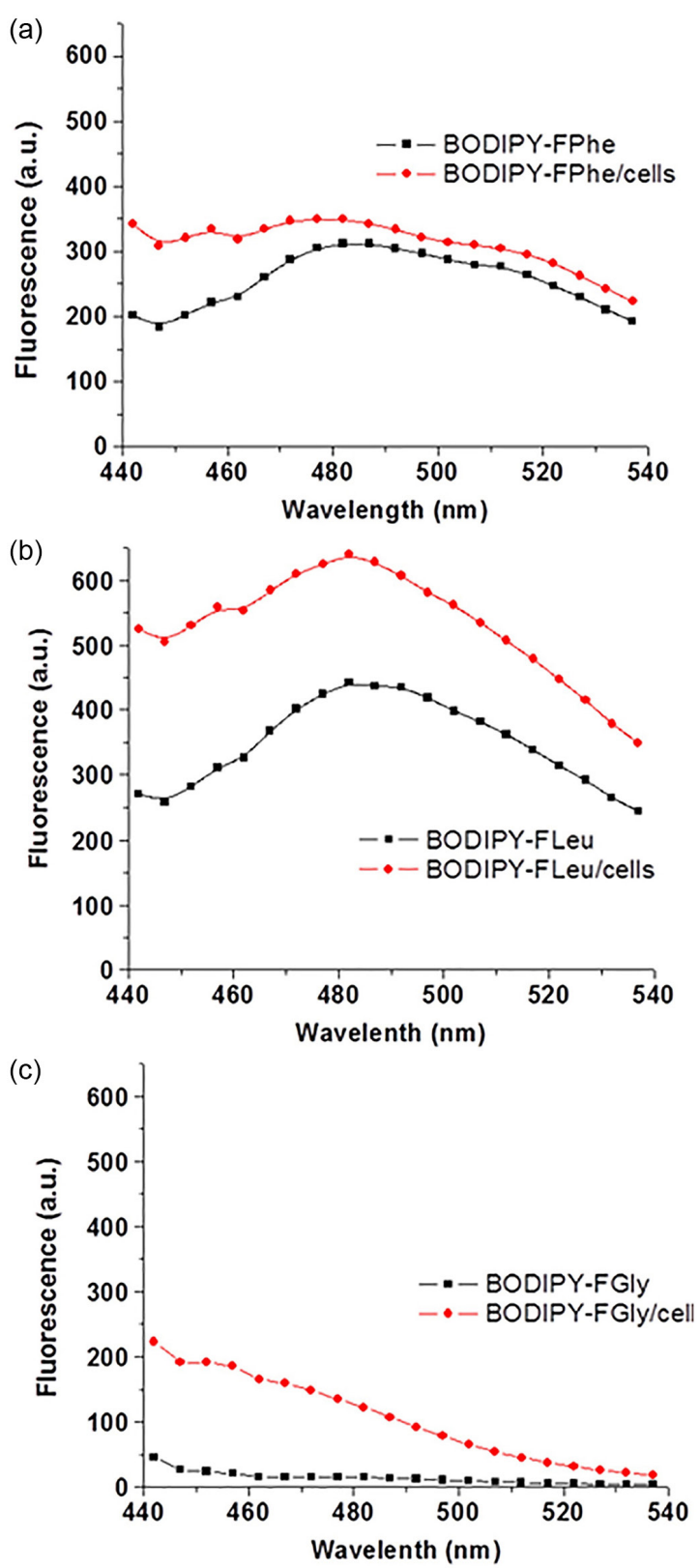

Figure 4. Fluorescence spectra of BODIPY-amino acid conjugates before and after internalization to hepatocytes cultured in suspension. outstanding contrast upon treatment of the cells with Hoechst (employed as a counterstain for cell nuclei, blue channel). However, illumination of the cells with a selected, constant intensity (1\%) of the green-channel laser $(488 \mathrm{~nm})$ revealed BODIPY-FLeu as the sample rendering the best results, in good agreement with the outcome obtained at suspension conditions. Interestingly, this superior performance of BODIPY-FLeu is not related to its quantum yield given that it is not the highest attained (see Table 1). As mentioned before, it appears that there is selectivity for leucine by cellular components of the membrane and the cytoplasm. These probes did not stain the nuclei in either case.

To broaden the characterization of BODIPY-FLeu on $\beta$-cells physiology, now we treated cell cultures with conditions that promote cellular stress (Figure 6). In a first approach, we evaluated the effect of tunicamycin (Tum), an inhibitor of the protein $\mathrm{N}$-glycosylation process. ${ }^{4}$ This condition triggers a condition denominated ER stress, associated with dysfunction of $\beta$-cells. Likewise, we evaluated the effect of lipotoxicity induced by palmitic acid (PA), wherein the capacity of protein folding may be compromised. ${ }^{45}$ Under these conditions, results obtained with the use of BODIPY-FLeu suggest a differential localization of the probe (Figure 6). In cells treated with Tum, the intensity of the BODIPY-FLeu signal (Figures 6c, 6d) was reduced as compared to controls (Figures 6a, 6b). Under Tum-treatment the maturation of proteins in the ER is affected, modifying protein homeostasis. ${ }^{3}$ The accumulation of unfolded proteins likely interferes with BODIPY-FLeu localization. However, in PA treatment, the intensity was higher than controls (Figures 6e, 6f), which is possibly associated with the expansion of cellular membranes, although more experimentation is necessary.

In a complementary way, we performed experiments to determine the cellular localization of BODIPY-FLeu.

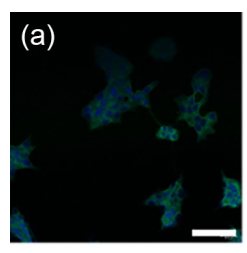

(b)

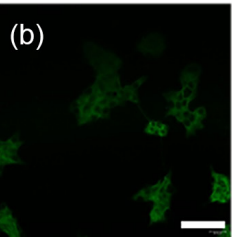

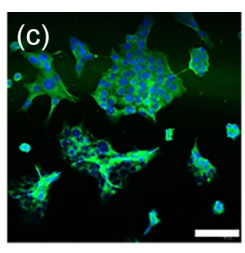

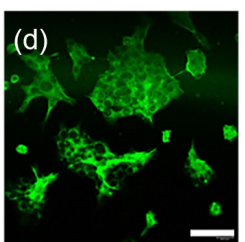

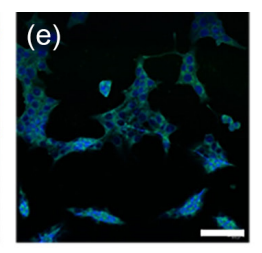

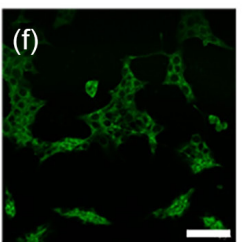

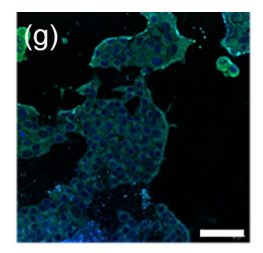

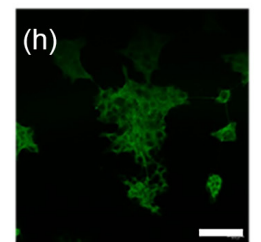

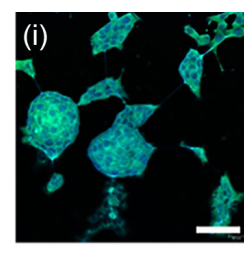

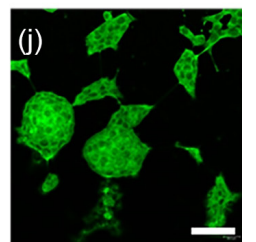

Figure 5. Images of $\beta$-cells treated with BODIPY-amino acid conjugates. (a, b) BODIPY-FGly; (c, d) BODIPY-FLeu; (e, f) BODIPY-FPhe; $(\mathrm{g}, \mathrm{h})$ BODIPY-FTyr(trt); and $(\mathrm{i}, \mathrm{j})$ BODIPY-FAla. Cells in the top panels were visualized with the Hoechst probe for nuclei detection. Scale bars correspond to $50 \mu \mathrm{m}$. 

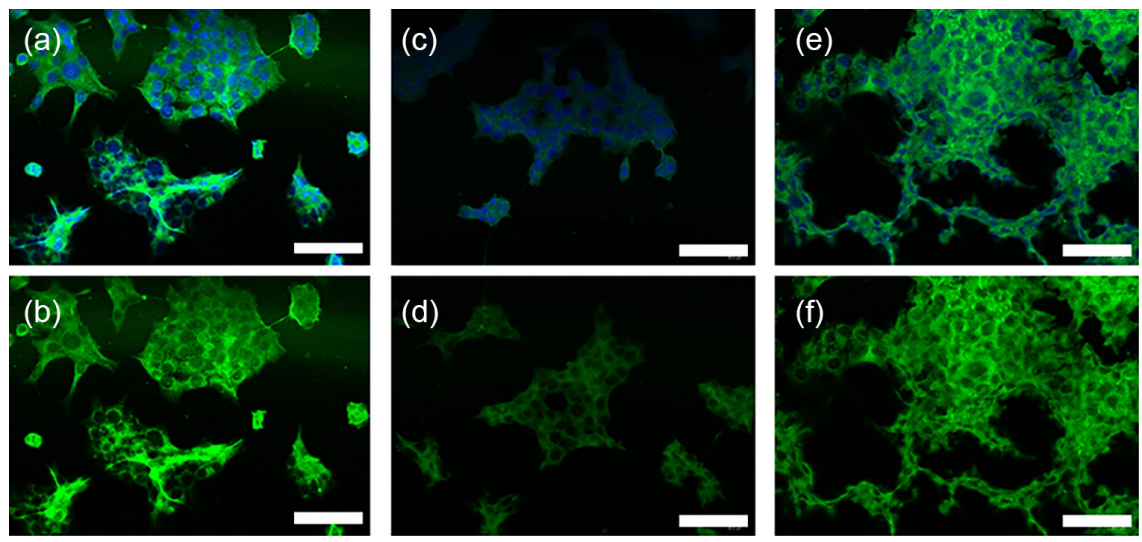

Figure 6. Effect of deleterious conditions in Langerhans $\beta$-cells on the localization of BODIPY-FLeu. (a, b) Control cells; (c, d) cells treated with tunicamycin $\left(2 \mu \mathrm{g} \mathrm{mL}^{-1}\right)$; (e, f) cells treated with PA $(300 \mu \mathrm{M})$. Cells in the top panels were visualized with the Hoechst probe for nuclei detection. Scale bars correspond to $50 \mu \mathrm{m}$.
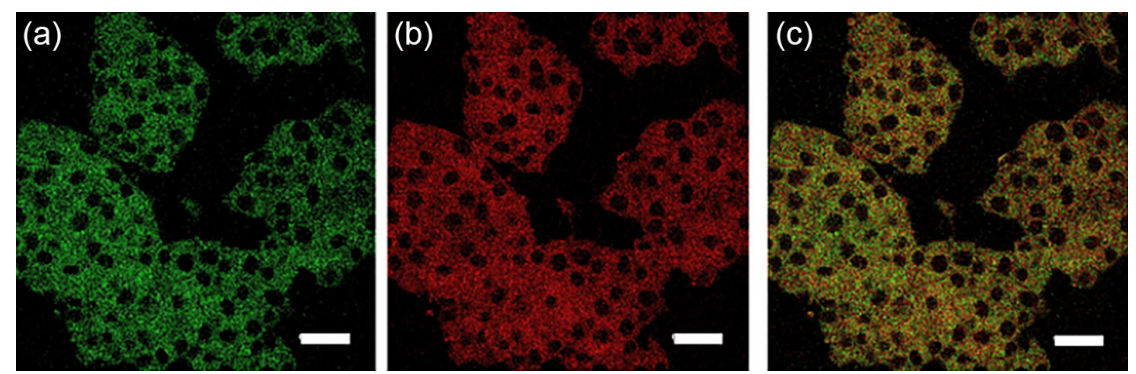

Figure 7. PS-BODIPY-FLeu colocalizes with ER-tracker. RIN-m5F cells were treated with PS-BODIPY-FLeu vesicles (a) and incubated with the ERtracker probe (b). (c) Image corresponds to the merge. Scale bars correspond to $25 \mu \mathrm{m}$.

Therefore, we generated lipid vesicles composed of phosphatidylserine (PS) $(30 \mu \mathrm{M})$ and BODIPY-FLeu $(6 \mu \mathrm{M})$ by ultra-sonication and cycles of freezing/thawing. Then, cell cultures of $\beta$-cells were treated with PS-BODIPY-FLeu vesicles, results suggest that PS-BODIPY-FLeu vesicles are internalized in RIN-m5F cells (Figure 7a), and BODIPY-FLeu could co-localize on ER (ER-tracker was used to co-localize ER sites; Figures 7b, 7c).

\section{Conclusions}

The coupling of BODIPY to amino acids glycine, alanine, leucine, phenylalanine, and tyrosine was carried out and the resulting conjugates were characterized in terms of their photophysical properties and cell staining functionality in a model of $\beta$-cells. Concerning their photophysical properties, all synthesized conjugates exhibited satisfactory levels of fluorescence emission; however, those containing amino acids with aromatic groups were found to display a higher optical efficiency. In particular, BODIPY-FTyr(trt) showed the highest coefficient of molar extinction and quantum yield. On the other hand, for their biological activity, the synthesized conjugates demonstrated three attractive features worth stressing. First, they had an outstanding selectivity for the cytoplasm region of suspension and adherent cells, being BODIPY-FLeu the one with the highest staining efficiency. Second, when dissolved in water, the conjugates presented a slight bathochromic shift of the absorption band, opening the possibility of acquiring confocal micrographs at both the blue and green channels on demand. Third, the localization of BODIPY-FLeu inside cells was found to be dependent on the cell state, originating a difference in intensity depending on ER stress induced by tunicamycin or lipotoxicity mediated by PA. Taken together, the obtained results highlight the synthesized conjugates as versatile cell staining agents with high selectivity for the cytoplasm of suspension and adherent cells, demonstrating not only a dual fluorescence emission (blue and green) but also a sensing capability to recognize different cell states.

\section{Supplementary Information}

Supplementary data (FTIR, NMR, and MS spectra) are available free of charge at $h t t p: / / j b c s . s b q . o r g . b r$ as a PDF file.

\section{Acknowledgments}

We gratefully acknowledge the support for this project 
by Consejo Nacional de Ciencia y Tecnología (CONACyT, grant No. 242823) and graduate scholarship (No. 221455), also acknowledge Tecnológico Nacional de México for the support to this project (Clave 7814.20-P). M.A.-M. thanks funding from CONACyT (Mexico) through Research Projects INFR-2015-251863 and PDCPN-2015-89.

\section{References}

1. Chen, X.; Wu, Y.-W.; Org. Biomol. Chem. 2016, 14, 5417.

2. Sticht, H.; Bayer, P.; Willbold, D.; Dames, S.; Hilbich, C.; Beyreuther, K.; Frank, R. W.; Rösch, P.; Eur. J. Biochem. 1995, 233, 293.

3. Díaz-Villanueva, F. J.; Díaz-Molina, R.; García-González, V.; Int. J. Mol. Sci. 2015, 16, 17193.

4. Savage, K. E.; Baur, P. S.; J. Cell Sci. 1983, 64, 295.

5. Strunz, U. T.; Walsh, J. H.; Grossman, M. I.; Proc. Soc. Exp. Biol. Med. 1978, 157, 440.

6. Isenberg, J. I.; Maxwell, V.; N. Engl. J. Med. 1978, 298, 27.

7. McArthur, K. E.; Isenberg, J. I.; Hogan, D. L.; Dreier, S. J.; J. Clin. Invest. 1983, 71, 1254.

8. Wang, C. C.; Grossman, M. I.; Am. J. Physiol.-Legacy Content 1951, 164, 527.

9. Meyer, J. H.; Grossman, M. I.; Am. J. Physiol.-Legacy Content 1972, 222, 1058.

10. Conigrave, A. D.; Quinn, S. J.; Brown, E. M.; Proc. Natl. Acad. Sci. U. S. A. 2000, 97, 4814.

11. Wang, S.; Tsun, Z.-Y.; Wolfson, R. L.; Shen, K.; Wyant, G. A.; Plovanich, M. E.; Yuan, E. D.; Jones, T. D.; Chantranupong, L.; Comb, W.; Wang, T.; Bar-Peled, L.; Zoncu, R.; Straub, C.; Kim, C.; Park, J.; Sabatini, B. L.; Sabatini, D. M.; Science 2015, 347, 188.

12. Rebsamen, M.; Pochini, L.; Stasyk, T.; de Araújo, M. E. G.; Galluccio, M.; Kandasamy, R. K.; Snijder, B.; Fauster, A.; Rudashevskaya, E. L.; Bruckner, M.; Scorzoni, S.; Filipek, P. A.; Huber, K. V. M.; Bigenzahn, J. W.; Heinz, L. X.; Kraft, C.; Bennett, K. L.; Indiveri, C.; Huber, L. A.; Superti-Furga, G.; Nature 2015, 519, 477.

13. Minami, T.; Esipenko, N. A.; Zhang, B.; Isaacs, L.; Anzenbacher, P.; Chem. Commun. 2014, 50, 61.

14. Debia, N. P.; Saraiva, M. T.; Martins, B. S.; Beal, R.; Gonçalves, P. F. B.; Rodembusch, F. S.; Alves, D.; Lüdtke, D. S.; J. Org. Chem. 2018, 83, 1348.

15. Debia, N. P.; Rodríguez, J. J. P.; da Silveira, C. H.; Chaves, O. A.; Iglesias, B. A.; Rodembusch, F. S.; Lüdtke, D. S.; J. Mol. Liq. 2020, 309, 113092.

16. Tao, K.; Levin, A.; Adler-Abramovich, L.; Gazit, E.; Chem. Soc. Rev. 2016, 45, 3935.

17. Kim, D.; Ma, D.; Kim, M.; Jung, Y.; Kim, N. H.; Lee, C.; Cho, S. W.; Park, S.; Huh, Y.; Jung, J.; Ahn, K. H.; J. Fluoresc. 2017, 27, 2231.
18. Mendive-Tapia, L.; Subiros-Funosas, R.; Zhao, C.; Albericio, F.; Read, N. D.; Lavilla, R.; Vendrell, M.; Nat. Protoc. 2017, 12, 1588 .

19. Pu, L.; Chem. Rev. 2004, 104, 1687.

20. Sinkeldam, R. W.; Greco, N. J.; Tor, Y.; Chem. Rev. 2010, 110, 2579.

21. Koyama, G.; Nakamura, H.; Muraoka, Y.; Takita, T.; Maeda, K.; Umezawa, H.; Iitaka, Y.; Tetrahedron Lett. 1968, 9, 4635.

22. Kang, J.; Huo, F.; Zhang, Y.; Chao, J.; Strongin, R. M.; Yin, C.; Sens. Actuators, B 2018, 273, 1532.

23. Kang, J.; Huo, F.; Yue, Y.; Wen, Y.; Chao, J.; Zhang, Y.; Yin, C.; Dyes Pigm. 2017, 136, 852.

24. Kang, J.; Huo, F.; Chao, J.; Yin, C.; Spectrochim. Acta, Part A 2018, 195, 16.

25. Kang, J.; Huo, F.; Ning, P.; Meng, X.; Chao, J.; Yin, C.; Sens. Actuators, B 2017, 250, 342.

26. Gómez-Durán, C. F. A.; García-Moreno, I.; Costela, A.; Martin, V.; Sastre, R.; Bañuelos, J.; López Arbeloa, F.; López Arbeloa, I.; Peña-Cabrera, E.; Chem. Commun. 2010, 46, 5103.

27. Loudet, A.; Burgess, K.; Chem. Rev. 2007, 107, 4891.

28. Goud, T. V.; Tutar, A.; Biellmann, J.-F.; Tetrahedron 2006, 62, 5084.

29. Ma, D. H.; Kim, D.; Akisawa, T.; Lee, K.-H.; Kim, K.-T.; Ahn, K. H.; Chem.-Asian J. 2015, 10, 894.

30. Wang, Y.; Pan, F.; Zhang, Y.; Peng, F.; Huang, Z.; Zhang, W.; Zhao, W.; Analyst 2016, 141, 4789.

31. Zhang, Y.; Peng, A.; Jie, X.; Lv, Y.; Wang, X.; Tian, Z.; ACS Appl. Mater. Interfaces 2017, 9, 13920.

32. Boens, N.; Verbelen, B.; Ortiz, M. J.; Jiao, L.; Dehaen, W.; Coord. Chem. Rev. 2019, 399, 213024.

33. Kowada, T.; Maeda, H.; Kikuchi, K.; Chem. Soc. Rev. 2015, $44,4953$.

34. Liu, Z.; Jiang, Z.; Yan, M.; Wang, X.; Front. Chem. 2019, 7, 712.

35. Kue, C. S.; Ng, S. Y.; Voon, S. H.; Kamkaew, A.; Chung, L. Y.; Kiew, L. V.; Lee, H. B.; Photochem. Photobiol. Sci. 2018, 17, 1691.

36. García-González, V.; Mas-Oliva, J.; Biochem. Biophys. Res. Commun. 2013, 434, 54.

37. García-González, V.; Gutiérrez-Quintanar, N.; Mas-Oliva, J.; Sci. Rep. 2015, 5, 16091.

38. Kyte, J.; Doolittle, R. F.; J. Mol. Biol. 1982, 157, 105.

39. Gustavsson, T.; Sarkar, N.; Lazzarotto, E.; Markovitsi, D.; Improta, R.; Chem. Phys. Lett. 2006, 429, 551.

40. Badea, M. G.; DeToma, R. P.; Brand, L.; Biophys. J. 1978, 24 , 197.

41. Garcia-Gonzalez, V.; Gutierrez-Quintanar, N.; MendozaEspinosa, P.; Brocos, P.; Pineiro, A.; Mas-Oliva, J.; J. Struct. Biol. 2014, 186, 19.

42. Pulido-Capiz, A.; Díaz-Molina, R.; Martínez-Navarro, I.; Guevara-Olaya, L. A.; Casanueva-Pérez, E.; Mas-Oliva, J.; 
Rivero, I. A.; García-González, V.; Front. Endocrinol. 2018, 9, 331 .

43. Acosta-Montano, P.; Garcia-Gonzalez, V.; Nutrients 2018, 10, 393.

44. Acosta-Montano, P.; Rodriguez-Velazquez, E.; Ibarra-Lopez, E.; Frayde-Gomez, H.; Mas-Oliva, J.; Delgado-Coello, B.; Rivero, I. A.; Alatorre-Meda, M.; Aguilera, J.; Guevara-Olaya, L.; Garcia-Gonzalez, V.; Cells 2019, 8, 884.
45. Galindo-Hernández, O.; Córdova-Guerrero, I.; Díaz-Rubio, L. J.; Pulido-Capiz, Á.; Díaz-Villanueva, J. F.; CastañedaSánchez, C. Y.; Serafín-Higuera, N.; García-González, V.; J. Cell. Biochem. 2019, 120, 4158.

Submitted: May 6, 2021

Published online: August 10, 2021 